\title{
5.0. DEPLETION, ACTIVATION, AND SPENT FUEL SOURCE TERMS
}

\section{Introduction by W. A. Wieselquist}

SCALE's general depletion, activation, and spent fuel source terms analysis capabilities are enabled through a family of modules related to the main ORIGEN depletion/irradiation/decay solver. The nuclide tracking in ORIGEN is based on the principle of explicitly modeling all available nuclides and transitions in the current fundamental nuclear data for decay and neutron-induced transmutation and relies on fundamental cross section and decay data in ENDF/B VII. Cross section data for materials and reaction processes not available in ENDF/B-VII are obtained from the JEFF-3.0/A special purpose European activation library containing 774 materials and 23 reaction channels with 12,617 neutroninduced reactions below $20 \mathrm{MeV}$. Resonance cross section corrections in the resolved and unresolved range are performed using a continuous-energy treatment by data modules in SCALE. All nuclear decay data, fission product yields, and gamma-ray emission data are developed from ENDF/B-VII.1 evaluations. Decay data include all ground and metastable state nuclides with half-lives greater than 1 millisecond. Using these data sources, ORIGEN currently tracks 174 actinides, 1149 fission products, and 974 activation products.

The purpose of this chapter is to describe the stand-alone capabilities and underlying methodology of ORIGEN_as opposed to the integrated depletion capability it provides in all coupled neutron transport/depletion sequences in SCALE, as described in other chapters. Through the stand-alone capabilities, there is generality to handle arbitrary systems (e.g., fast reactor fuel depletion or structural activation) by providing arbitrary flux spectra and arbitrary one-group cross sections to the module COUPLE, which in turn creates ORIGEN library (.f33) files containing the problem-dependent, onegroup reaction coefficients required to solve the actual equations governing depletion/decay. These libraries are required input for the ORIGEN module, along with the initial isotopics and irradiation history, in terms of either a time-dependent power or flux level. Two high-performance equation solvers are available: the hybrid linear chains and matrix exponential method and a new Chebyshev Rational Approximation Method (CRAM). Typical execution times are on the order of a few seconds for a multi-step solution, with each individual solution (step) taking approximately 10 milliseconds. ORIGEN also includes capabilities for continuous feed and removal by element. Output capabilities include isotopics (moles or grams), source spectra (alpha, beta, gamma, and neutron), activity (becquerels or curies), decay heat (total watts or gamma only), and radiological hazard factors (maximum permissible concentrations in air or water). These results can be displayed in the output file (.out extension) and/or archived in an ORIGEN binary results file (.f71 extension).

The use of current, fundamental data resources is a key feature of ORIGEN, including nuclear decay data, multigroup neutron reaction cross sections, neutron-induced fission product yields, and decay emission data for photons, neutrons, alpha particles, and beta particles. The nuclear decay data are based primarily on ENDF/B-VII.1 evaluations. The multigroup nuclear reaction cross section libraries now include evaluations from the JEFF-3.0/A neutron activation file containing data for 774 target nuclides, more than 12,000 neutron-induced reactions, and more than 20 different reaction types below $20 \mathrm{MeV}$, provided in various energy group structures. Energy-dependent ENDF/B-VII.0-based fission product yields are available for 30 fissionable actinides. Gamma-ray and x-ray emission data libraries are based on ENDF/B-VII.1. The photon libraries contain discrete photon line energy and intensity data for decay gamma-ray and $\mathrm{x}$-rays emission for 1,132 radionuclides, prompt and delayed continuum spectra for spontaneous fission, $(\alpha, n)$ reactions in oxide fuel, and bremsstrahlung from decay beta (electron and positron) particles slowing down in either a $\mathrm{UO}_{2}$ fuel or water matrix. Methods and data libraries used to calculate the neutron yields and energy spectra for spontaneous fission, $(\alpha, n)$ reactions, and delayed neutron emission are adopted from the SOURCES4C code. Capabilities to calculate the beta and alpha particle emission source and spectra have also been added. 
The ORIGEN reactor libraries distributed with SCALE include a set of pre-calculated ORIGEN libraries (with TRITON) for a variety of fuel assembly designs:

- $\quad$ BWR 7×7, 8×8-1, 8×8-2, 9×9-2, 9×9-9, 10×10-9, 10×10-8, SVEA-64, SVEA-96, and SVEA-100;

- $\quad$ PWR $14 \times 14,15 \times 15,16 \times 16,17 \times 17,18 \times 18$;

- $\quad$ CANDU reactor (19-, 28-, and 37-element bundle designs);

- $\quad$ Magnox graphite reactor;

- $\quad$ Advanced Gas-Cooled Reactor (AGR);

- $\quad$ VVER 440 and VVER 1000;

- RBMK;

- IRT;

- $\quad$ MOX BWR 7×7, 8×8-1, 8×8-2, 9×9-2, 9×9-9, 10×10-9, 10×10-8, SVEA-64, SVEA-96, and SVEA-100;

- $\quad$ MOX PWR 14×14, 15×15, 16×16, 17×17, 18×18.

These libraries may be used to rapidly assess spent fuel isotopics and source terms in these systems for arbitrary burnups and decay times. For $\mathrm{UO}_{2}$-based assembly isotopics, the new ORIGAMI sequence provides a very convenient, easy-to-use interface. The most general capability, and requiring more user input, is available using the ARP interpolator module in conjunction with the ORIGEN solver module.

Finally, with regards to user interfaces, ORIGEN has a new keyword-based input in SCALE 6.2 but also maintains the ability to read SCALE 6.1 input. Both ORIGEN and ORIGAMI are tightly integrated with the SCALE graphical user interface, Fulcrum, which includes syntax highlighting, input checking with immediate feedback, and (.f71) output viewing. Additionally, Fulcrum provides an ORIGAMI Automator project interface to characterize the fuel inventory for an entire reactor site and generate data needed for severe accident analysis. ORIGAMI Automator is not documented in this chapter, but a primer is available with step by step instructions on its use. 